EPJ Web of Conferences 108, 02046 (2016)

DOI: $10.1051 /$ epjconf/201610802046

CC Owned by the authors, published by EDP Sciences, 2016

\title{
Scattering Problem and Resonances for Three-Body Coulomb Quantum Systems: Parallel Calculations
}

\author{
E. Yarevsky ${ }^{1, a}$ \\ ${ }^{1}$ Department of Computational Physics, St Petersburg State University, St Petersburg, Russia
}

\begin{abstract}
An approach to the solution of scattering and resonance problems based on splitting the potential into a finite range part and a long range tail part is proposed. The explicit solution to the Schrödinger equation for the long range tail Hamiltonian is used as an incoming wave. This reformulation of the scattering problem makes it suitable for treatment by the exterior complex scaling. The same technique is used to determine resonances of the system. Calculations are performed with the finite element method which allows efficient parallel computations. The approach is illustrated with calculations of the electron resonant scattering on the hydrogen and the helium ion.
\end{abstract}

\section{Introduction}

The study of scattering processes in few-body systems is a difficult task from both theoretical and computational points of view. This complexity originates from intricate asymptotic boundary conditions for the wave function at large distances [1]. These boundary conditions are especially complicated if the long-range Coulomb interaction is involved. For such systems, methods which allow solving the problem without explicit use of the asymptotic form of the wave function are of big importance.

Several approaches are based on the complex scaling method [2]. Originally, this technique was based on the uniform rotation of coordinates [3] and could only be applied to problems involving exponentially decreasing potentials. Later, this method was modified in such a way that some longer range potentials could be studied [4]. Essentially, the modification consisted in replacing the potential $V(r)$ by a finite cut potential $V_{R}(r)=V(r)$, for $r<R$, and $V_{R}(r)=0$, for $r \geq R$. As the potential $V_{R}(r)$ is not an analytic function on the whole semiaxis, the exterior complex scaling (ECS) beyond the point $r=R$ was employed.

This modified approach cannot be applied directly to the scattering problem with the Coulomb interaction in the asymptotic configuration since the cut-off of the Coulomb potential at any $R$ distorts the asymptotic behavior of the solution at large separation of particles [1]. In recent papers [5-7] there has been developed a method accurately solving the Schrödinger equation for the Coulomb scattering problem using ECS. This approach is based on the sharp splitting of the Coulomb potential for constructing the distorted incident wave which is generated by the tail part of the Coulomb potential. This reformulation of the scattering problem makes it suitable for the application of the ECS technique.

a e-mail: e.yarevsky@spbu.ru 
In this paper, the application of the potential splitting approach to the electron scattering of the hydrogen and the positive helium ion is presented. This scattering problem is of fundamental importance for the quantum and atomic physics so any newly developed approach is worth testing on it. In Section 2, the equations of the potential splitting approach are derived. In Section 3, we discuss our numerical approach and results for the electron-hydrogen and electron- $\mathrm{He}^{+}$systems. Atomic units are used throughout.

\section{Theoretical approach}

In the centre-of-mass, a three quantum particle system is described by six-dimensional coordinates. The total angular momentum representation allows for the separation of the rotational motion so the Schrödinger equation is reduced to a finite system of coupled three-dimensional differential equations $[7,8]$. For the sake of simplicity, only zero angular momentum is considered here. In this case, the system of equations consists of one equation only. In the standard Jacobi coordinates $\mathbf{x}_{\alpha}, \mathbf{y}_{\alpha}$, it is written as

$$
(H-E) \Psi\left(x_{\alpha}, y_{\alpha}, \theta_{\alpha}\right)=\left(H^{K}+\sum_{\alpha=1}^{3} V_{\alpha}\left(x_{\alpha}\right)-E\right) \Psi\left(x_{\alpha}, y_{\alpha}, \theta_{\alpha}\right)=0,
$$

where $x_{\alpha}=\left|\mathbf{x}_{\alpha}\right|, y_{\alpha}=\left|\mathbf{y}_{\alpha}\right|, \theta_{\alpha}=\left(\mathbf{x}_{\alpha}, \mathbf{y}_{\alpha}\right) / x_{\alpha} y_{\alpha}, \alpha=1,2,3$. The total interaction is defined as the sum of two-body interactions $V\left(x_{\alpha}\right)=q_{\beta} q_{\gamma} / x_{\alpha}$. The kinetic energy is given by

$$
H^{K}=-\frac{1}{2 \mu_{\alpha}^{x}} \frac{\partial^{2}}{\partial x_{\alpha}^{2}}-\left\{\frac{1}{2 \mu_{\alpha}^{y} y_{\alpha}^{2}}+\frac{1}{2 \mu_{\alpha}^{x} x_{\alpha}^{2}}\right\}\left(\frac{\partial^{2}}{\partial \theta_{\alpha}{ }^{2}}+\cot \theta_{\alpha} \frac{\partial}{\partial \theta_{\alpha}}\right)-\frac{1}{2 \mu_{\alpha}^{y}} \frac{\partial^{2}}{\partial y_{\alpha}^{2}},
$$

where $\mu_{\alpha}^{x}, \mu_{\alpha}^{y}$ are the reduced masses [1].

In order to solve Eq. (1), one should supply it with boundary conditions. These are trivial for calculations of bound states and rather complicated for scattering processes, see below. System resonances can hardly be calculated without a transformation of Eq. (1).

For studying resonances, the complex scaling (rotation) method is used [2,8]. In this method, each of the spatial coordinates is replaced with the complex one $r \rightarrow s_{\phi}(r)$. Any function $u(r)$ is then transformed as $\left(W^{\phi} u\right)(r)=\sqrt{|J(\phi)|} u\left[s_{\phi}(r)\right]$, where $J(\phi)$ is the Jacobian $J(r)=d s_{\phi}(r) / d r$. The rotated Hamiltonian is expressed as $H(\phi)=W^{\phi} H\left(W^{\phi}\right)^{-1}$. The Hamiltonian $H(\phi)$ is complex symmetric but not self-adjoint. Hence it may have a complex spectrum, and the resonances result as the $\phi$ independent complex eigenvalues of this Hamiltonian.

\subsection{Potential splitting approach}

The solution of the scattering problem for Eq. (1) involves complicated boundary conditions [1]. They are hard to implement especially for the Coulomb interactions. So let us describe here in short the potential splitting approach [5-7] which allows solving the Coulomb scattering problem without explicit use of the asymptotic form of the wave function.

Let $\chi^{R}(r)$ be the indicator of the domain $r \geq R$, i.e.

$$
\chi^{R}(r)=\left\{\begin{array}{cc}
0, & r<R \\
1, & r \geq R
\end{array}\right.
$$

and $\chi_{R}=1-\chi^{R}$ be its complementary partner. The reaction potential $V^{\alpha}\left(x_{\alpha}, y_{\alpha}, \theta_{\alpha}\right)$,

$$
V^{\alpha}\left(x_{\alpha}, y_{\alpha}, \theta_{\alpha}\right)=\sum_{\beta=1}^{3} V_{\beta}\left(x_{\beta}\right)-V_{\alpha}\left(x_{\alpha}\right)=\sum_{\beta \neq \alpha} V_{\beta}\left(x_{\beta}\right)
$$


is split into the sum of the core, $V_{R}$, and the tail, $V^{R}$, parts

$$
V^{\alpha}\left(x_{\alpha}, y_{\alpha}, \theta_{\alpha}\right)=V_{R}\left(x_{\alpha}, y_{\alpha}, \theta_{\alpha}\right)+V^{R}\left(x_{\alpha}, y_{\alpha}, \theta_{\alpha}\right)
$$

where

$$
V_{R}=V^{\alpha} \chi_{R}\left(y_{\alpha}\right), \quad V^{R}=V^{\alpha} \chi^{R}\left(y_{\alpha}\right) .
$$

The distorted incident wave $\Psi^{R}\left(x_{\alpha}, y_{\alpha}, \theta_{\alpha}\right)$ is the solution to the scattering problem for the sum of $V_{\alpha}\left(x_{\alpha}\right)$ and the tail potential $V^{R}\left(x_{\alpha}, y_{\alpha}, \theta_{\alpha}\right)$

$$
\left[H^{K}+V_{\alpha}\left(x_{\alpha}\right)+V^{R}\left(x_{\alpha}, y_{\alpha}, \theta_{\alpha}\right)-E\right] \Psi^{R}\left(x_{\alpha}, y_{\alpha}, \theta_{\alpha}\right)=0 .
$$

For $\Phi \equiv \Psi-\Psi^{R}$ one has the driven Schrödinger equation

$$
(H-E) \Phi=-V_{R} \Psi^{R} .
$$

This is the main equation of the potential splitting approach. Its right hand side (r.h.s.) is of finite range with respect to the variable $y_{\alpha}$. Besides, $\Phi$ behaves as a superposition of pure outgoing waves in all asymptotic regions. Therefore, Eq. (8) is suitable for the exterior complex scaling with the exterior scaling radius $R_{\mathrm{ECS}} \geq R$. After ECS, $\Phi$ becomes exponentially decreasing function so the zero boundary conditions can be used in order to solve the rotated equation (8).

Let us now discuss the construction of the incident wave $\Psi^{R}$. The tail potential $V^{R}$ is replaced with its leading term in the incident configuration $V_{C}^{R}\left(y_{\alpha}\right)=q_{\alpha}\left(q_{\beta}+q_{\gamma}\right) y_{\alpha}^{-1} \chi^{R}\left(y_{\alpha}\right)$. The variables can be separated for the potential $V_{C}^{R}$ :

$$
\Psi_{0}^{R}\left(\mathbf{x}_{\alpha}, \mathbf{y}_{\alpha}\right)=\varphi_{i}\left(\mathbf{x}_{\alpha}\right) \psi_{C}^{R}\left(\mathbf{y}_{\alpha}, \mathbf{p}_{i}\right),
$$

where $\mathbf{p}_{i}$ is the incoming momentum. The function $\varphi_{i}\left(\mathbf{x}_{\alpha}\right)=x_{\alpha}^{-1} R_{n_{i}, l_{i}}\left(x_{\alpha}\right) Y_{\ell_{i}, m_{i}}\left(\hat{x}_{\alpha}\right)$ is the target bound state wave function for the two-body system. The function $\psi_{C}^{R}\left(\mathbf{y}_{\alpha}, \mathbf{p}_{i}\right)$ can be constructed explicitly [7]. In the region $\left(y_{\alpha} \leq R\right)$ it is given by

$$
\psi_{C}^{R}\left(\mathbf{y}_{\alpha}\right)=\frac{4 \pi}{p_{i} y_{\alpha}} \sum_{\ell, m} a_{\ell}^{R} i^{\ell} \hat{j}_{\ell}\left(p_{i} y_{\alpha}\right) Y_{\ell, m}\left(\hat{p}_{i}\right) Y_{\ell, m}\left(\hat{y}_{\alpha}\right),
$$

where

$$
a_{\ell}^{R}=e^{i \sigma_{\ell}} W_{R}\left(F_{\ell}, u_{\ell}^{+}\right) / W_{R}\left(\hat{j}_{\ell}, u_{\ell}^{+}\right) .
$$

Here $\hat{j}_{\ell}$ is the Riccati-Bessel function, and $\sigma_{\ell}$ is the Coulomb phase shift. The function

$$
u_{\ell}^{+}\left(\eta_{i}, p_{i} y_{\alpha}\right)=e^{-i \sigma_{\ell}}\left(G_{\ell}\left(\eta_{i}, p_{i} y_{\alpha}\right)+i F_{\ell}\left(\eta_{i}, p_{i} y_{\alpha}\right)\right)
$$

is expressed in terms of the regular $F_{\ell}$ and irregular $G_{\ell}$ Coulomb wave functions. The Sommerfeld parameter $\eta_{i}=\mu_{\alpha}^{y} q_{\alpha}\left(q_{\beta}+q_{\gamma}\right) / p_{i}$ and the Wronskian $W_{R}(f, g)=f(r) g^{\prime}(r)-f^{\prime}(r) g(r)$ are calculated at $r=R$.

The function $\Psi_{0}^{R}(9)$ does not satisfy Eq. (7) exactly, $\Psi^{R} \neq \Psi_{0}^{R}$. However, $\Psi_{0}^{R}$ approaches $\Psi^{R}$ when $R \rightarrow \infty$. Indeed, in the region where $\varphi_{i}\left(\mathbf{x}_{\alpha}\right)$ is not negligible one obtains

$$
V^{R}\left(x_{\alpha}, y_{\alpha}, \theta_{\alpha}\right)-V_{C}^{R}\left(y_{\alpha}\right) \sim O\left(y_{\alpha}^{-2}\right) .
$$

So the difference of the potentials has a non-Coulomb tail and can be truncated as in already developed applications of ECS [4]. As in the truncation region $y_{\alpha} \geq R$, the difference (and the approximation error) goes to zero when $R$ goes to infinity. More arguments on this subject can be found in papers [6, 7] but the conclusive proof (like one in the two body case [5]) still remains to be done. 


\subsection{Asymptotic behavior of the scattering wave function}

On solving (8) with ECS, we obtain the wave function $\Phi$ in the region $x_{\alpha}, y_{\alpha} \leq R$. The next step is to calculate the amplitudes and cross sections corresponding to the various scattering processes occurring in the system. The methods of amplitude calculations are based on the asymptotic form of the wave function at large distances. Below the ionization threshold, this form reads [1]

$$
\Phi\left(x_{\alpha}, y_{\alpha}, \theta_{\alpha}\right) \sim \sum_{n, \ell} \tilde{A}_{n, \ell} \frac{R_{n, \ell}\left(x_{\alpha}\right)}{x_{\alpha}} u_{\ell}^{+}\left(\eta_{n}, p_{n} y_{\alpha}\right) Y_{\ell, 0}\left(\theta_{\alpha}, 0\right) .
$$

The full state-to-state scattering amplitude $A_{n, \ell}=\tilde{A}_{n, \ell}+A_{n, \ell}^{R}$ is expressed in terms of $A_{n, \ell}^{R}$ which is calculated explicitly using the representation of $\psi_{C}^{R}$ for $y_{\alpha}>R$. By projecting (12) on the two body wave functions, we get the local representation for the partial amplitudes $\tilde{A}_{n, \ell}$ for large $y_{\alpha}$

$$
\tilde{A}_{n, \ell} \approx \frac{\left(u_{\ell}^{+}\left(\eta_{n}, p_{n} y_{\alpha}\right)\right)^{-1}}{2 \pi} \int_{0}^{\infty} d x_{\alpha} \int_{0}^{\pi} \sin \theta_{\alpha} d \theta_{\alpha} x_{\alpha} R_{n, \ell}\left(x_{\alpha}\right) \Phi\left(x_{\alpha}, y_{\alpha}, \theta_{\alpha}\right) Y_{\ell, 0}\left(\theta_{\alpha}, 0\right) .
$$

Thereby the solution of the scattering problem becomes a two-stage process. First, the exterior complex rotation with $R_{\mathrm{ECS}} \geq R$ is applied to the driven equation (8):

$$
(H(\phi)-E)\left(W^{\phi} \Phi\right)=-W^{\phi} V_{R} \Psi^{R} .
$$

Both the solution $W^{\phi} \Phi$ and the r.h.s. decrease at infinity so this equation is to be solved with the zero boundary conditions at infinity. Second, the scattering amplitudes are calculated from the nonrotated spatial part of the solution, i.e. $x_{\alpha}, y_{\alpha} \leq R_{\mathrm{ECS}}$, with representation (13). This means that the asymptotic behavior (12) is used so $R_{\mathrm{ECS}}$ should be chosen large enough to secure solution validity.

\section{Numerical method and results}

In order to demonstrate how our approach works, the electron- $\mathrm{H}$ and electron- $\mathrm{He}^{+}$systems have been chosen. The theoretical study of these systems was done in many reports (see $[9,10]$ and references therein) using different approximations and methods. It is assumed here that the nucleus has an infinite mass so the reduced masses $\mu_{\alpha}^{x}=\mu_{\alpha}^{y}=1$, and the distances $x_{\alpha}, y_{\alpha}$ are the electron-nucleus distances. No peculiarities appear in the equations due to this assumption so they do not need any special modification. As the electrons are identical fermions, the wave function should be properly symmetrized with respect to the permutation of the electron coordinates. The permutation operator commutes with the Hamiltonian $H(\phi)$ so the r.h.s. of Eq. (14) only should be symmetrized.

In order to solve numerically (14) for the scattering problem and its homogeneous counterpart for the resonances, the finite element method (FEM) has been employed $[8,11]$. In the calculations, we use a rectangular grid formed by the same one-dimensional grid in both coordinates $x_{\alpha}$ and $y_{\alpha}$. For each coordinate, we use five finite elements at short distance [0-4] a.u., and four elements of total length 40 a.u. for the discretization beyond the rotation point $R$. The intermediate region [4-91] a.u. is divided into elements with a length 3 a.u. One element only was used for the angular variable $\theta_{\alpha}$.

Details of our computational realization of the FEM can be found in $[8,11]$. Here we just stress that the most of the time is consumed by computations of the matrix elements of the Hamiltonian and the r.h.s. vector in Eq. (14). So these were parallelized in our code. For the FEM, the natural choice is the distribution of the finite elements among different processors using the MPI technique [11]. The 


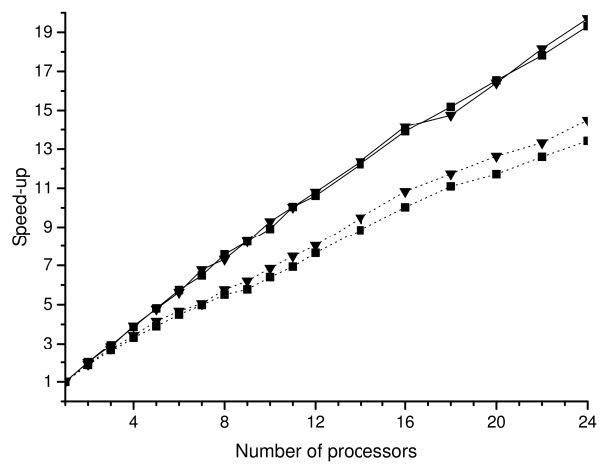

Figure 1. Calculation speed-up as a function of the number of processors. The squares and triangles are the results for the FEM polynomial degree 7 and 9, respectively. The solid line corresponds to the matrix elements of the Hamiltonian in Eq. (14), and the dot line does to its right hand side.
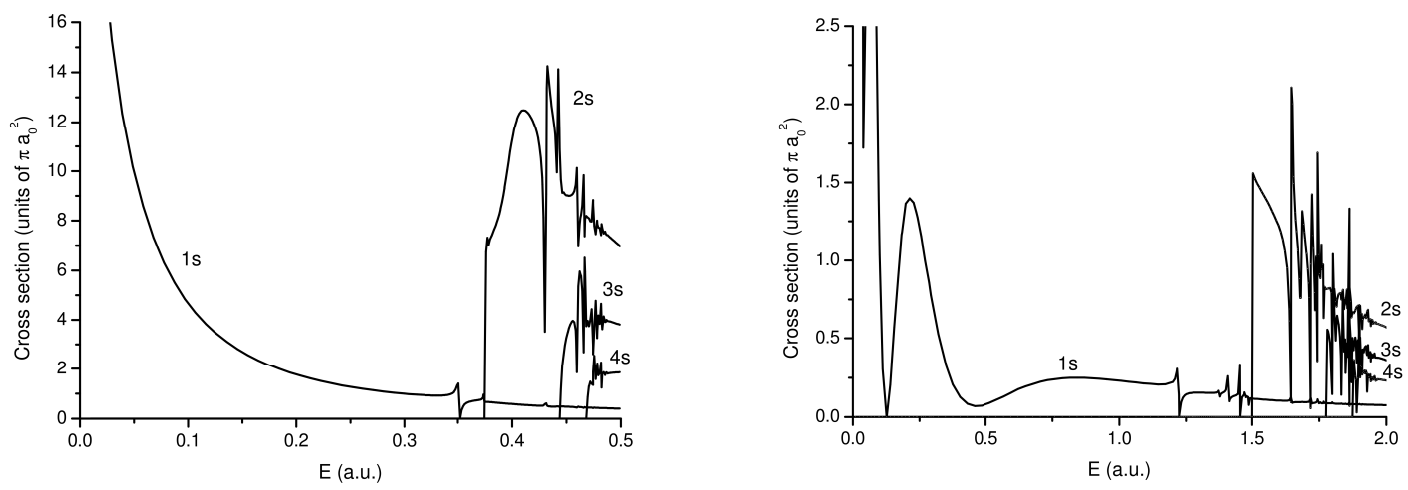

Figure 2. The singlet (spin weight included) $1 \mathrm{~s} \rightarrow 1 \mathrm{~s}$ elastic and $1 \mathrm{~s} \rightarrow 2 \mathrm{~s}, 3 \mathrm{~s}, 4 \mathrm{~s}$ excitation cross sections for the $\mathrm{e}-\mathrm{H}(l e f t)$ and $\mathrm{e}-\mathrm{He}^{+}(r i g h t)$ scattering as a function of the incident electron energy. The values are multiplied by 200,400 , and 600 for $2 \mathrm{~s}, 3 \mathrm{~s}$, and $4 \mathrm{~s}$ cross sections, respectively.

presented results have been obtained with the Intel Cluster Studio 2013. The program has been run on HP ProLiant DL980 G7 server based on Intel Xeon X7560 processors at 2.2 GHz.

Figure 1 shows the calculation speed-up as a function of the number of processors. The calculation of the matrix elements of the Hamiltonian takes more than $90 \%$ of the total time for a given energy and can be speed up to 19 times. Changing of the polynomial degree (i.e. the number of the matrix elements on each finite element) has little influence on the speed-up while the computation time changes substantially. The speed-up achieved for the r.h.s. computation is smaller as the ratio of the data processed to the data transferred is smaller for the vector compare to the matrix.

On figure 2 our results for the singlet $1 \mathrm{~s} \rightarrow \mathrm{ns}$ cross sections for the e- $\mathrm{H}$ and e- $\mathrm{He}^{+}$scattering are presented. The cross sections have very rich resonance structure especially the e- $\mathrm{He}^{+}$cross section due to the asymptotic Coulomb interaction. Due to this interaction the cross section exhibits also oscillating behavior at small energies. The calculated values were compared to other results [10, 12, 13]. The relative difference was found to be less than $10^{-3}$. As the cross section structure is so complicated, it is very important to have an independent tool to analyze this structure. In our approach it is possible to calculate resonances independently with the ECS method. The energies of few Helium resonances are given in table 1 . These values agree very well with previous calculations [8] that have been carefully optimized to get accurate resonance energies. They also perfectly match peculiarities in the scattering data on figure 2. 
Table 1. Few deepest resonances of He atom below the second threshold

$$
\begin{aligned}
& 1.22214 \text {-i } 2.27(-3) \\
& 1.37839 \text {-i } 1.09(-4) \\
& 1.39742 \text {-i } 3.33(-6) \\
& 1.41011 \text {-i } 6.83(-4) \\
& 1.44026 \text {-i } 1.30(-7) \\
& 1.45195 \text {-i } 3.82(-5)
\end{aligned}
$$

\section{Conclusions}

Mathematically sound approach for calculations of both resonances and scattering processes is presented. Potential splitting method allows for the solution of the scattering problem with the Coulomb interaction. The use of the unified approach minimizes numerical uncertainties and facilitates comparison of the scattering and resonance data. Comparison of the scattering data and resonances in multichannel systems cannot rely on resonance energies only. For example, influence of resonance states on cross sections has been analyzed in terms of S-matrix residues for the two-body two-channel model of the charge transfer reaction $\mathrm{N}^{3+}+\mathrm{H} \rightarrow \mathrm{NH}^{3+} \rightarrow \mathrm{N}^{2+}+\mathrm{H}^{+}$[14]. The generalization of that technique for three-body systems remains to be done.

\section{Acknowledgements}

This work is supported by the Russian Foundation for Basic Research grant No. 14-02-00326 and by St Petersburg State University within the project No. 11.38.241.2015. Calculations were partially performed using the facilities of the "Computational Center of SPbS".

\section{References}

[1] L. D. Faddeev, S. P. Merkuriev, Quantum Scattering Theory for Several Particle Systems (Dordrecht: Kluwer, 1993)

[2] E. Balslev, J. M. Combes, Commun. Math. Phys. 22, 280-294 (1971)

[3] J. Nuttall, H. L. Cohen, Phys. Rev. 188, 1542-1543 (1969)

[4] T. N. Rescigno, M. Baertschy, D. Byrum, C. W. McCurdy, Phys. Rev. A 55, 4253 (1997)

[5] M. V. Volkov, S. L. Yakovlev, E. A. Yarevsky, N. Elander, Phys. Rev. A 83, 032722 (2011)

[6] E. Yarevsky, S. L. Yakovlev, Å. Larson, N. Elander, J. Phys. B. 48, 115002 (2015)

[7] M. V. Volkov, E. A. Yarevsky, S. L. Yakovlev, Europhysics Letters 110, 30006 (2015)

[8] N. Elander, S. Levin, E. Yarevsky, Phys. Rev. A 67, 062508 (2003)

[9] R. J. W. Henry, Phys. Rep. 68, 1-91 (1981)

[10] P. L. Bartlett, J. Phys. B: At. Mol. Opt. Phys. 39, R379-R424 (2006)

[11] E. Yarevsky, LNCS 7125: Mathematical Modeling and Computational Science (Springer, 2012) 290-295

[12] J. Botero, J. Shertzer, Phys. Rev. A 46, R1155-R1158 (1992)

[13] Y. D. Wang, J. Callaway, Phys. Rev. A 48, 2058-2069 (1993)

[14] K. Shilyaeva, N. Elander, E. Yarevsky, J. Phys. B. 42, 044011 (2009) 